\title{
Every Library's Nightmare? Digital Rights Management, Use Restrictions, and Licensed Scholarly Digital Resources ${ }^{1}$
}

\section{Kristin R. Eschenfelder}

This study explored use restrictions found in licensed scholarly resources from the fields of history/art history, engineering, and health sciences. The analysis developed a framework of use restrictions that distinguishes between soft restrictions-which discourage use-and hard restrictions-which strictly prevent use. Soft restrictions include: extent of use, obfuscation, omission, amalgamation, frustration, and warning. The study concludes that these soft restrictions are relatively common in licensed scholarly resources. Further, while hard restrictions are less common, they are not unknown. The study questions whether librarians should be doing more to challenge use restrictions.

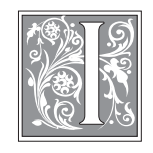

$n$ recent years librarians have grown increasingly concerned that vendors of licensed scholarly resources (e.g., e-journals, e-books) will put digital rights management tools or "technological protection measures" (TPM) on the resources licensed by academic libraries. TPM are configurations of hardware and software used to control access to, or use of, a digital work by restricting particular uses such as saving or printing. ${ }^{2}$ Librarian concerns about TPM stem not only from the aggressive implementation of TPM by the movie, music, and popular e-book industries, but also from recent academic e-book vendor experimentation with TPM.

The question of whether or not TPM are necessary to protect digital works in a networked environment applies in many industries, including academic publishing. Within publishing, some have argued TPM are necessary to convince content owners to make their works digitally available. For example, NetLibrary advertises its "one page at a time viewing" DRM as a form of protection to lure potential content contributors to offer their books on the NetLibrary platform. ${ }^{3}$ Others have argued that the academic publishing community is unlikely to adopt TPM because academic publishing business models are built on library subscriptions that are unlikely to change due to uncontrolled use by individual library patrons. ${ }^{4,5}$ Further, many objectionable uses (such as unauthorized spidering of vendor servers or downloads of entire journal runs) are already controlled through established protocols between

Kristin R. Eschenfelder is Associate Professor in the School of Library and Information Studies at the University of Wisconsin-Madison; e-mail: eschenfelder@wisc.edu. 
publishers and libraries employing license terms, server monitoring systems, and disabling of offending IP numbers followed up by campus level disciplinary action and education of users. ${ }^{6}$ Given stable subscription rates and functioning protocols to deal with abuses, vendors may be unwilling to pay for TPM, or reconfigure already developed proprietary interfaces to accommodate TPM. ${ }^{7}$

Within the academic library community, concerns about vendor use of TPM are complicated by already existing interface or delivery platform designs that, to some extent, determine the possible uses of their information products. These design decisions directly affect users' satisfaction with and uses of information products. ${ }^{8}$ As one participant in a digital library usability study asked, "...can the user print, save, and e-mail the desired information? If the user can't 'take' the information in the form that they want, it has a negative impact on them." ${ }^{\prime 9}$

While usability studies have noted problems with use restrictions, more attention has been paid to what this paper refers to as "hard restrictions," or the secure-container TPM more common in the movie and music industries. Many have passionately argued that these TPM will undermine libraries' mission of preserving and providing access to knowledge, erode fair use rights, ${ }^{10}$ and reduce innovation by limiting how information can be used and who can participate in the creation of new works. ${ }^{11}$ Others are concerned that vendors can use TPM to support use-based, or "pay per view," pricing models. By restricting saving or printing of digital works, downloads of these works and vendors' revenues will increase. Describing the new pricing possibilities afforded by TPM, one librarian worried: "What may be every publisher/vendor's dream could be every library's nightmare."12 Library advocacy organizations argue that TPM may create user dissatisfaction, generate interoperability problems, block archival and preservation activities, and require increased staffing to handle support and training requirements. ${ }^{13}$

While TPM are clearly cause for concern, this paper argues that academic librarians may have overlooked the use restrictions already common in licensed scholarly resources. This paper describes the output of a study to explore the use restrictions currently found in scholarly information products licensed by academic libraries. Use restrictions explored include limits on use activities such as printing, saving, and e-mailing. ${ }^{14}$ This paper seeks to answer three questions: (1) What use restrictions do authorized users of licensed scholarly resources experience? (2) Are these restrictions addressed in vendors' acceptable use statements? (3) To what extent do these use restrictions qualify as TPM? The study examined use restrictions present in samples of licensed resources from engineering, health sciences, and history/art history.

This paper employs a critical information studies (CIS) stance. CIS seeks to reveal the structures and practices that channel flows of information and cultural elements, and the processes by which legal outcomes unfold..$^{15}$ The study results inform larger questions about the prevalence of TPM use in the scholarly resource marketplace, and it raises new questions about librarians' expectations about use restrictions in the products they select and license. Results show that vendor use of the strictly limiting TPM employed in the movie and music industries is relatively rare. But many vendor products do contain a range of use-discouraging features or "soft restrictions." While soft restrictions do not strictly prevent uses, they discourage certain uses by making them difficult to enact.

While these soft restrictions do not garner the attention that TPM do, they are also problematic. Beyond the fact that they may discourage use, they may also become so taken-for-granted that librarians and users accept them as unassailable fact, or part of the natural e-resources order. ${ }^{16}$ Scholars have argued that the 
long-term importance of TPM is that they change expectations of, or awareness about, what one ought to be able to do with digital works. ${ }^{17}$ This paper suggests that the soft restrictions that are present in licensed products may have already changed users' and librarians' expectations about what use rights they ought to expect from vendors and their products. Given this possibility, it is important to clarify what use restrictions are currently in place and what use restrictions librarians may already have come to take for granted as part of the natural order of licensed digital products.

\section{Background}

Anxieties about TPM, and use restrictions more broadly, stem from the increasing reliance of academic libraries on content that is (a) digital, and (b) licensed. The digital nature of resources is significant because digital formats facilitate imposition of TPM - or what Lessig calls "code-based" use controls. ${ }^{18}$ Further, as Lessig explains, because digital use necessarily involves making a copy of a work (i.e., downloading a document from the vendor's server), rights holders argue that they can place greater restrictions on use of that copy than they could place on a paper work used multiple times. ${ }^{19}$ Moreover, because digital materials are vendor hosted, vendors can make changes on the host server-such as implementation of TPM - without guaranteeing the technical cooperation of libraries.

The licensed nature of much academic library material is also relevant. Importantly, the license provides some protection from TPM. Because a license defines the terms and conditions under which a digital work is offered, vendors would need to offer a new license that detailed changes in use terms to implement a TPM. And, libraries would need to agree to that license..$^{20}$ Some, however, have expressed concern that vendor introduction of TPM will reduce libraries' ability to negotiate favorable use terms in licenses. As vendors build restrictions into TPM, these restrictions could bound what uses are considered negotiable. For example, vendors could argue that certain use restrictions are inherent in the technology platforms, and therefore unavoidable. Building restrictions into delivery platforms may make them more difficult to negotiate away. ${ }^{21}$ Another concern is that the code-based restrictions of TPM take away the cushion of vagueness permitted by licenses. The imprecise nature of license language often leaves rights not clearly forbidden as arguably permitted. TPM, however, could block any uses not clearly allowed by the license. As Coyle explains, with TPM any right that is not specified is not given. Widespread implementation of TPM could require that librarians specify all possible uses during license negotiations. ${ }^{22}$

\section{A Use-based Conception of Use Restrictions}

To illuminate the types of use restrictions libraries may already take for granted, the study employed an inductive approach to defining use restrictions. Instead of only looking for examples of resources with the hard restriction, secure-container TPM problematized in the literature, we investigated any use restriction an authorized user would experience in "typical scholarly use" of a licensed resource. ${ }^{23}$

"Typical scholarly use" was defined in terms of use actions, extent of use, current and subsequent use, and individual/group use. Use actions included copy, paste, save, print, and e-mail. ${ }^{24}$ Extent of use included number of pages, number of articles, and duration of use..$^{25}$ In this study, extent of use was conservative so as to not violate license agreements. Current versus subsequent use was determined by whether or not a file could be saved in an easily re-accessible form for potential later use. ${ }^{26}$ Distinction between individual and group use reflects concerns about sharing rights among scholars (or students) given the growing collaborative nature of research ${ }^{27}$ Collaboration potential was assessed by whether or not the work, or 


\begin{tabular}{|l|l|}
\multicolumn{1}{|c|}{ TABLE 1 } \\
\multicolumn{1}{|c|}{ A User View of Use Restrictions } \\
\hline \hline $\begin{array}{l}\text { Soft } \\
\text { Restrictions }\end{array}$ & $\begin{array}{l}\text { Interface or server side configurations of software or hardware that may } \\
\text { discourage certain uses such as saving, printing multiple pages, e-mailing. } \\
\text { Importantly, the desired use may be achieved through workarounds such } \\
\text { as multiple sessions, or operating system or browser functionalities. These } \\
\text { workarounds may not be obvious, and they may involve inconvenience to } \\
\text { the user. }\end{array}$ \\
\hline $\begin{array}{l}\text { Hard } \\
\text { Restrictions }\end{array}$ & $\begin{array}{l}\text { Systems that strictly prevent uses such as saving, printing, or e-mailing } \\
\text { despite operating system or browser functionalities. }\end{array}$ \\
\hline
\end{tabular}

a useful representation, could easily be attached to an e-mail.

Pretesting quickly led us to the distinction between restrictions that made certain uses inconvenient, and restrictions that strictly prevented certain uses. The paper refers to the former as "soft" restrictions and the latter as "hard" restrictions. Soft restrictions are configurations of hardware or software that make certain uses such as printing, saving, copy/pasting or e-mailing more difficult-but not impossible-to achieve. Arguably, soft restrictions still limit use by making them very inconvenient.

Hard restrictions are configurations of software or hardware that strictly prevent certain uses. Hard restrictions include, but are not limited to, secure-container TPM systems that encrypt content and require an external software device to decrypt and serve the content to the user. ${ }^{28}$ The software device may simply be a plug-in to existing popular content readers like Adobe Acrobat Reader or Microsoft Media Player. In these cases, if the plug in is pre-installed, the user might not notice the presence of the TPM until desired use is blocked. In other instances, secure container systems require custom applications that the user must download to view the content (for example, the Overdrive e-book media player). In these cases, the specialized player makes it much more obvious that a hard TPM is in use.

\section{Methodology}

The goal of data collection was to document the types of use restrictions an authorized user experiences when making typical scholarly use of a sample of licensed scholarly digital resources. We also compared the observed restrictions with vendors' acceptable use statements. Analysis aimed to develop a use-based framework of restrictions based on the observed restrictions.

Data collection was conducted at one Carnegie I research institution with a large collection of electronic resources from spring-fall 2006. The research included resources from three subject areas: engineering, health sciences, and history/ art history. Data collection involved three stages: resource sampling, use rights assessment and interviews with librarians. While the data are from one site, the TPM observed are arguably similar to what might be seen at other universities with similar collections.

A combination of purposeful and random sampling was used to select resources for use rights assessment. ${ }^{29}$ Initial interviews with librarians from the target subject areas identified resources that might contain use restrictions. In addition, a random sample of 10 percent of resources was drawn from each discipline. Total samples included:

- Engineering-24 resources

- Health-27 resources

- History/Art History-26 resources $^{30}$ The resources in the sample included reference resources, full text article databases, e-book collections, historic letter, diary and newspaper collections, and collections of data or chemical structures. Vendors of these resources included both 
large and small for-profit and nonprofit organizations, and both primary publishers and aggregators.

The use rights assessment was performed using a scenario-based protocol that dictated how much of the resource was accessed, and how each resource was used. ${ }^{31}$ Because the assessment scenario employed very conservative use guidelines, it is possible that our assessment may not have uncovered higher-use threshold restrictions. ${ }^{32}$

Use rights restrictions were identified by comparing the experience of using the selected resource with presumed use rights drawn from the literature. Notes were made using a standardized form. Any inconsistencies were noted on the form and the resource was then retested. The resource is only reported here if the observed restriction could be recreated by two data collectors.

Presumed use rights: Users can view all of the selection. User can print the selection. User can copy and paste large portions of the selection (text and or graphics). User can save a copy of the selection to local disk. User can view the local copy, print the local copy, and e-mail the local copy.

Interviews included 14 librarians from the three subject areas. Interview data included identification of resources with use restrictions, stories of student and faculty problems with use restrictions, and descriptions of vendor activities.

Inductive data analysis generated a framework of use restriction types (see Table 2: Summary of Use Restriction Types and Examples from the Data). Analysis also compared the observed use restrictions to use limitations described in acceptable use statements (or equivalent) on vendor Web sites.

\section{Results: Observed use restrictions}

This section describes the outcome of data analysis: the framework of use-based restrictions including both soft and hard restrictions. For each, a definition and examples of subtypes from the data are presented. Each example includes discussion of the relevant terms of use as described by the vendor.

\section{Soft Restrictions}

As noted earlier, pretesting quickly distinguished between soft and hard restrictions. Soft restrictions are configurations of software or hardware that discourage certain uses. With soft restrictions, desired use may be ultimately achieved through workarounds; however, some of these workarounds may involve considerable inconvenience to the user. We found six subcategories of soft use restrictions outlined below. Many resources included combinations of these subcategories.

\section{Soft Type 1: Extent of Use}

The first restriction type, extent of use, is commonly employed by vendors on their servers to block "suspicious" or "excessive" patterns of use. But often vendors do not clearly define suspicious or excessive use patterns. For example, the NetLibrary e-book collection warns users: "If a suspicious usage pattern indicates excessive copying, the activity is logged and you are sent a copyright warning message." 33 Further, vendors' parameters for suspicious use may change over time based on customer feedback. For example, Blackwell Publishing announced an increase in its PDF download limit and apologized for inconvenience caused to libraries by the previously lower limit. ${ }^{34}$ Whether or not extent of use restrictions impede legitimate scholarly use would depend on the restriction and on the scholarly use. Restrictions may block use after a user saves too many articles from one issue of a journal; or, they may only kick in when a user attempts to download an entire journal corpus. The fact that Blackwell changed its download limit suggests that, at least in some instances, extent of use restrictions do impede legitimate scholarly use. 
Other extent of use examples clearly advertise their use limits. For example, several resources had print/save "batch size limits" that could interfere with some legitimate use. As shown in figure 1, the Eighteen Century Collections Online (ECCO) warns users of a view/print limit of 50 pages during any user session (see figure 1). Early English Books Online (EEBO) limits the number of record items in a marked citation list that can be printed or saved at one time (see figure 2 ), but no restrictions were found for printing/saving within texts. Early American Imprints warns users "Please note that a single download of multiple pages may consist of up to 25 pages." In another example, the CRC Handbook of Chemistry and Physics only permitted printing or saving of a maximum of 25 rows of data from any data table at one time. We classify these as soft restrictions because, in most cases, users can print or save beyond the limit by employing multiple separate batches.
These use restrictions are sometimes referred to in Acceptable Use statements; for example, ECCO states that it allows users to make print copies of "a permitted portion of the content" for fair use purposes. ${ }^{35}$ EEBO's use statement refers to potential restrictions on particular texts, "Individual content providers or licensors may have conditions of use applicable solely to their content." But it goes on to promise that the conditions "shall not materially alter your use of the Products." Librarian interviews, however, suggested that the observed restrictions in ECCO and EEBO caused problems for users whose research demanded a larger extent of use.

Soft Type 2: Restriction by Frustration When content is broken up into chunks, printing and saving portions of the content can become frustrating - but not impossible to accomplish. Chunking is a common practice in e-books and e-texts,

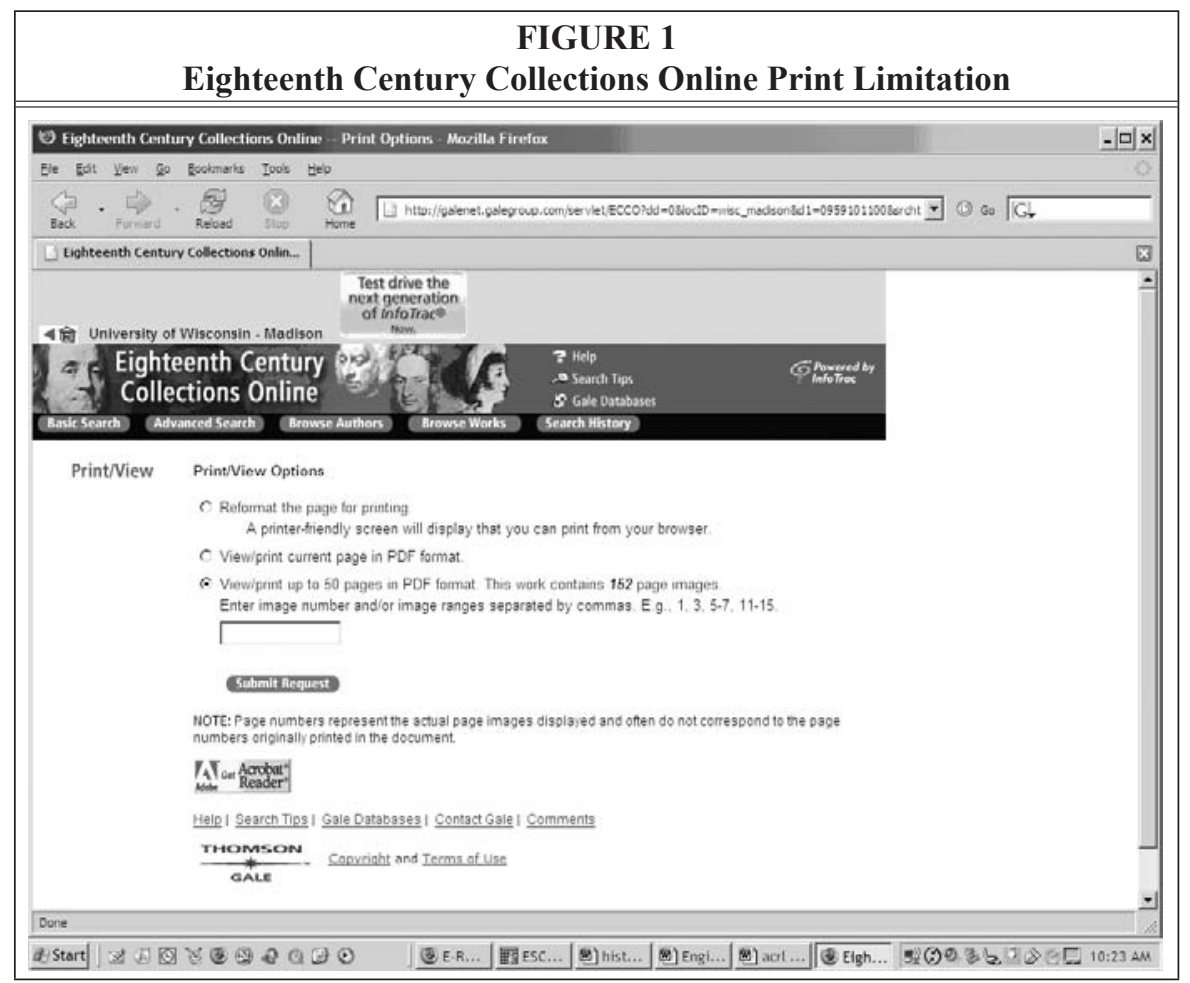




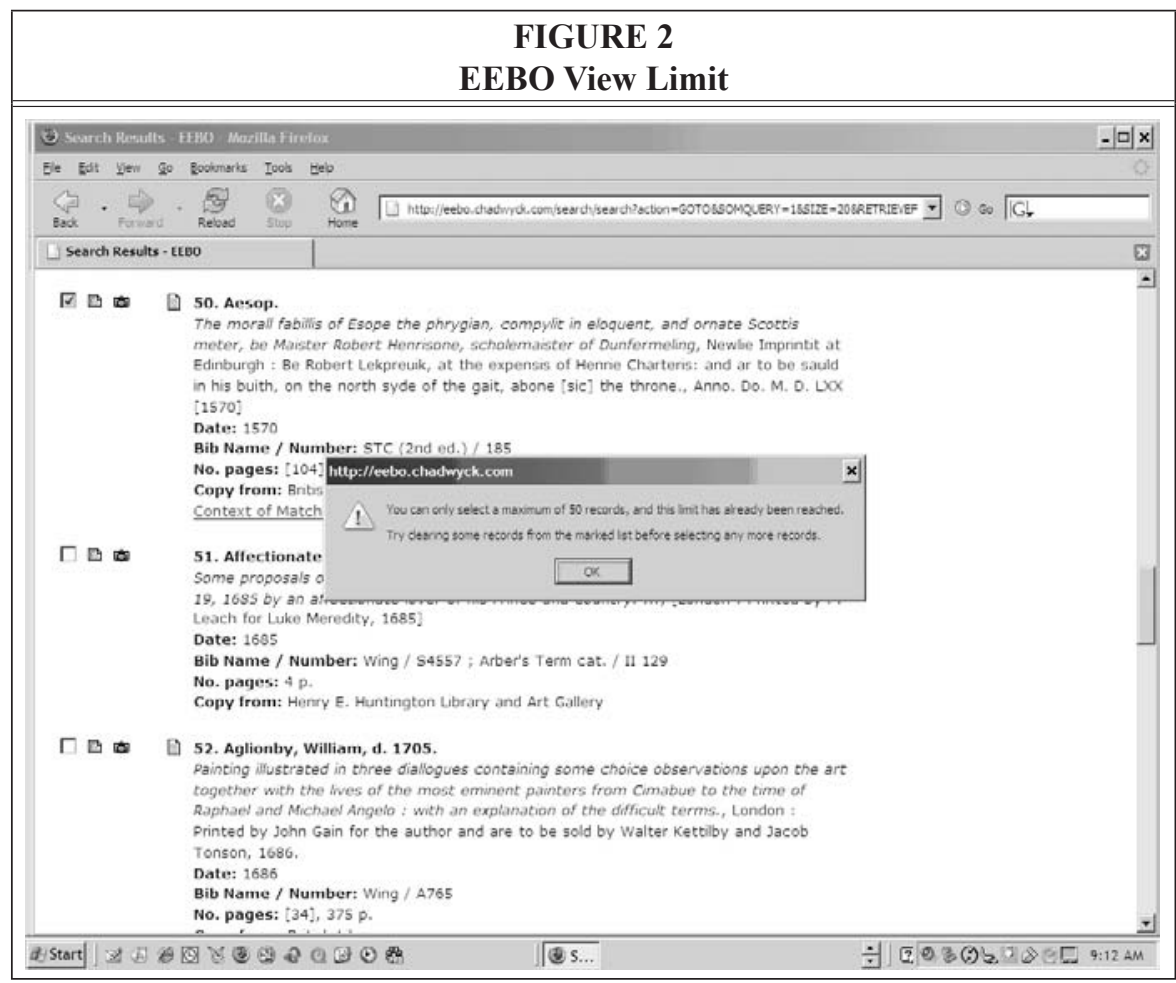

but the amount of content in a chunk varies by resource. Many e-book collections show only one page view in a chunk (for example, NetLibrary, shown in figure 3 -note page 1 of 1 shown in the Acrobat page indicator at bottom of screen) and the National Academy Press. In these situations, users must print or save one page at a time.

Other collections, such as EngNetBase books, will display an entire chapter as a chunk. Finally other resources, such as Referex, display the entire book as a chunk-including 676-page books (see figure $4-$ Note that all $17.7 \mathrm{MB}$ of 676 pages have downloaded in the Acrobat page indicator in the bottom center of the screen). While small chunks are notoriously unpopular, very large chunks are not always popular with users. Librarians explained that users complain when they are forced to download and save very large files as opposed to just the sections of content they are most interested in.
Most e-book acceptable use policies allow for saving and printing for personal or fair use purposes. ${ }^{36}$ Arguably, however, the personal or fair use referred to in their acceptable use statements suggests a more convenient personal/fair use than their interface allows.

\section{Soft Type 3: Obfuscation}

In some cases, poorly designed interfaces do not adequately advertise possible use functionalities (for instance, print, save), potentially leading some users to assume they cannot enact the desired use. For example, Early English Books Online (EBBO) provides liberal use functionality if one tags a record and then accesses it through a "marked list" link. But if the user does not tag records, functionality is limited to one page at a time access. Similarly, the Up To Date health sciences resource requires that users choose a "printer friendly" version before saving a complete document. Not choosing the printer-friendly version leads to a user saving only one page at 


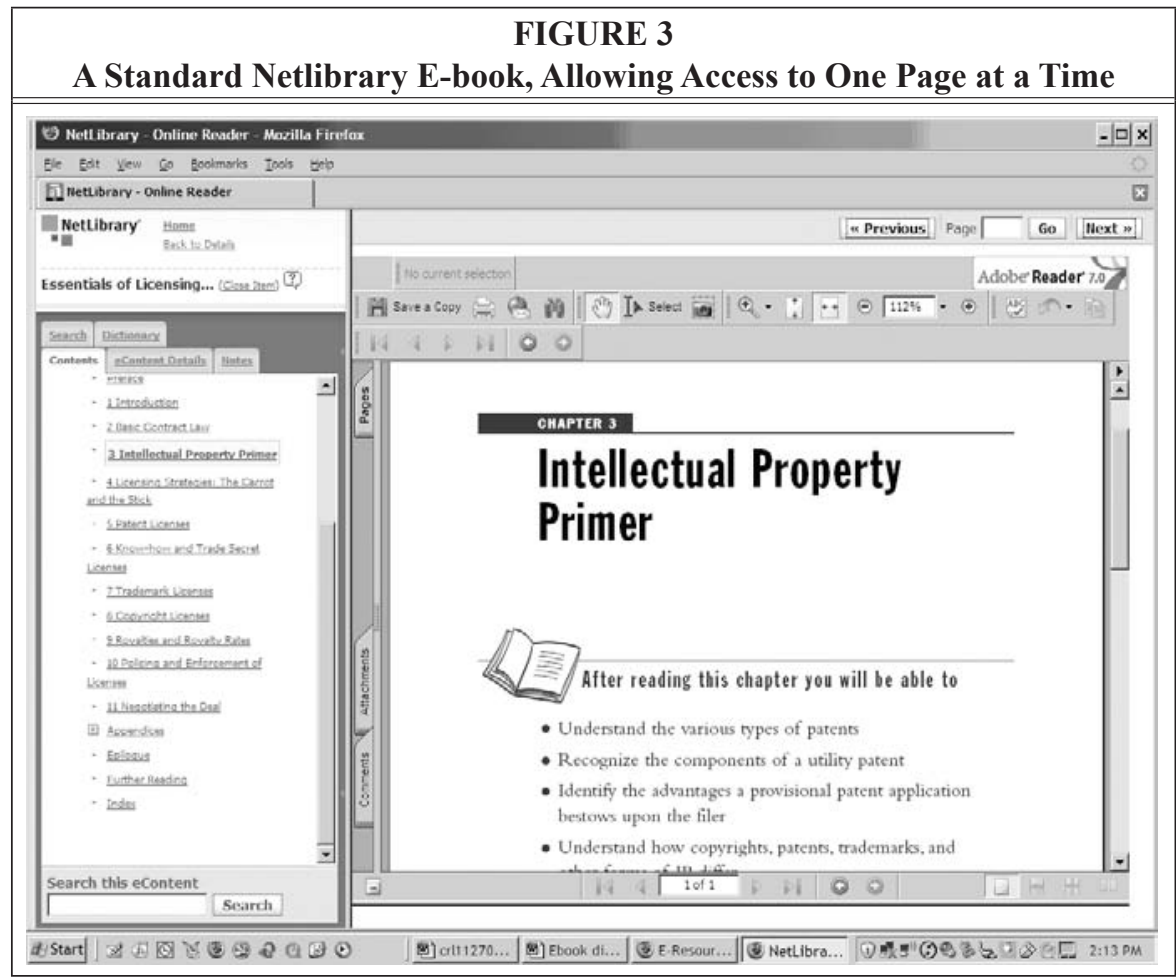

FIGURE 4

Referex Book Collection Displays the Entire 676 Pages of a Book at One Time

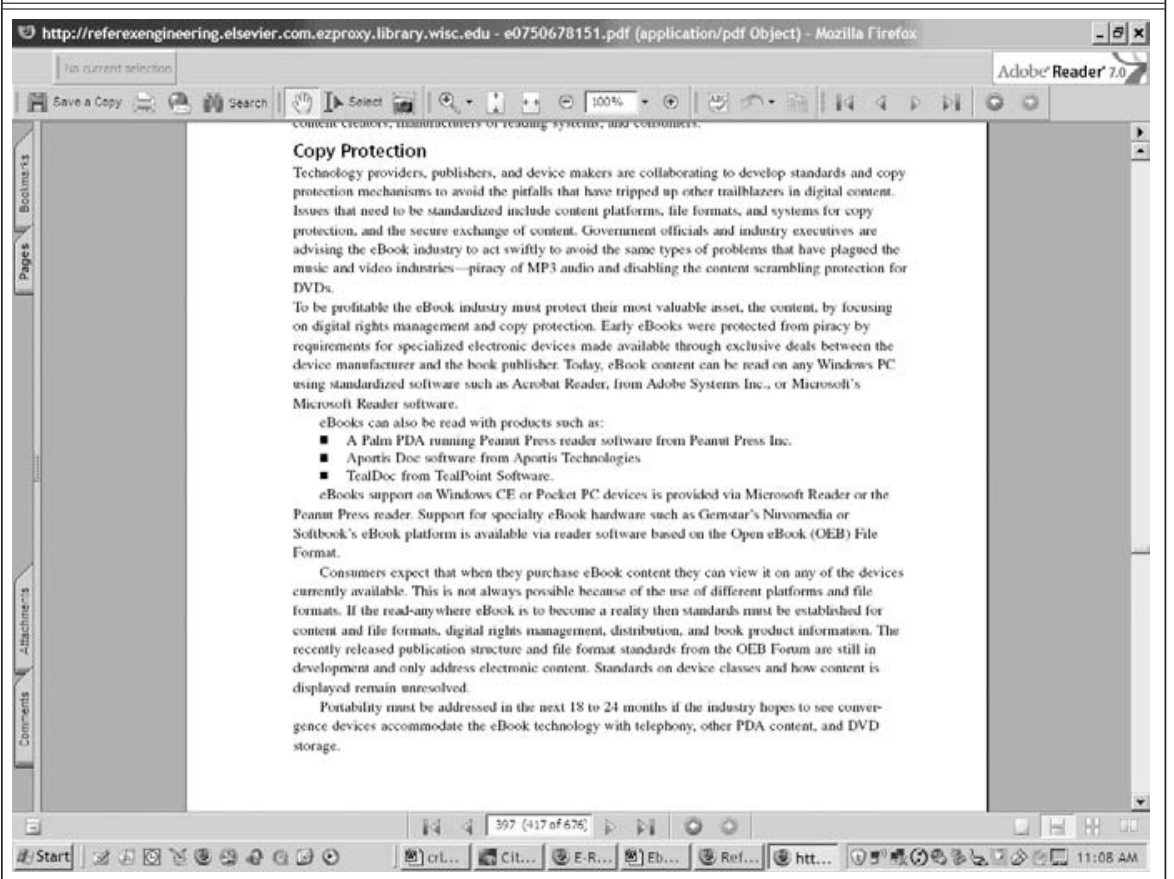


a time. Finally, in interviews, librarians noted that some novice users miss the multipage print and save links in JSTOR, and end up printing and saving one page image at a time. Arguably, these users are not getting all the use options promised them by the resources' acceptable use policies. The use statement may guarantee certain uses, but the interface makes the uses harder to realize.

\section{Soft Type 4: Interface Omission}

In these examples, certain use functionalities were not provided in the resource native interface and were only possible though the use of browser and/or computer operating system functions. Users who limited themselves to native interface features might assume that some desired uses are not possible. For example, numerous resources did not provide an "e-mail" button, but e-mailing was usually possible if the file was saved locally first. In another example, some HTML e-books in
NetLibrary have no copy or save buttons, and the user must employ tools from the browser or operating system to copy or save. Figure 5 shows an attempt to copy a text chunk from a NetLibrary book..$^{37}$ As shown, the interface does not include a copy button; further, the operating system right-click feature does not produce the expected "copy" option but, rather, a custom menu. ${ }^{38}$ It is possible to copy the text if the user employs the browser copy tool under the "Edit" menu. But this ability to copy is not obvious from examination of the interface or from right-clicking - indeed the author missed it and assumed copying was not possible. Thankfully, she was corrected by a research assistant double-checking the observation.

NetLibrary's Terms of Use statement explicitly gives permission to copy within the bounds of fair use. ${ }^{39}$ So while it is permissible to copy text from this e-book, the interface's omission of a specific copy feature, combined with its disabling of the

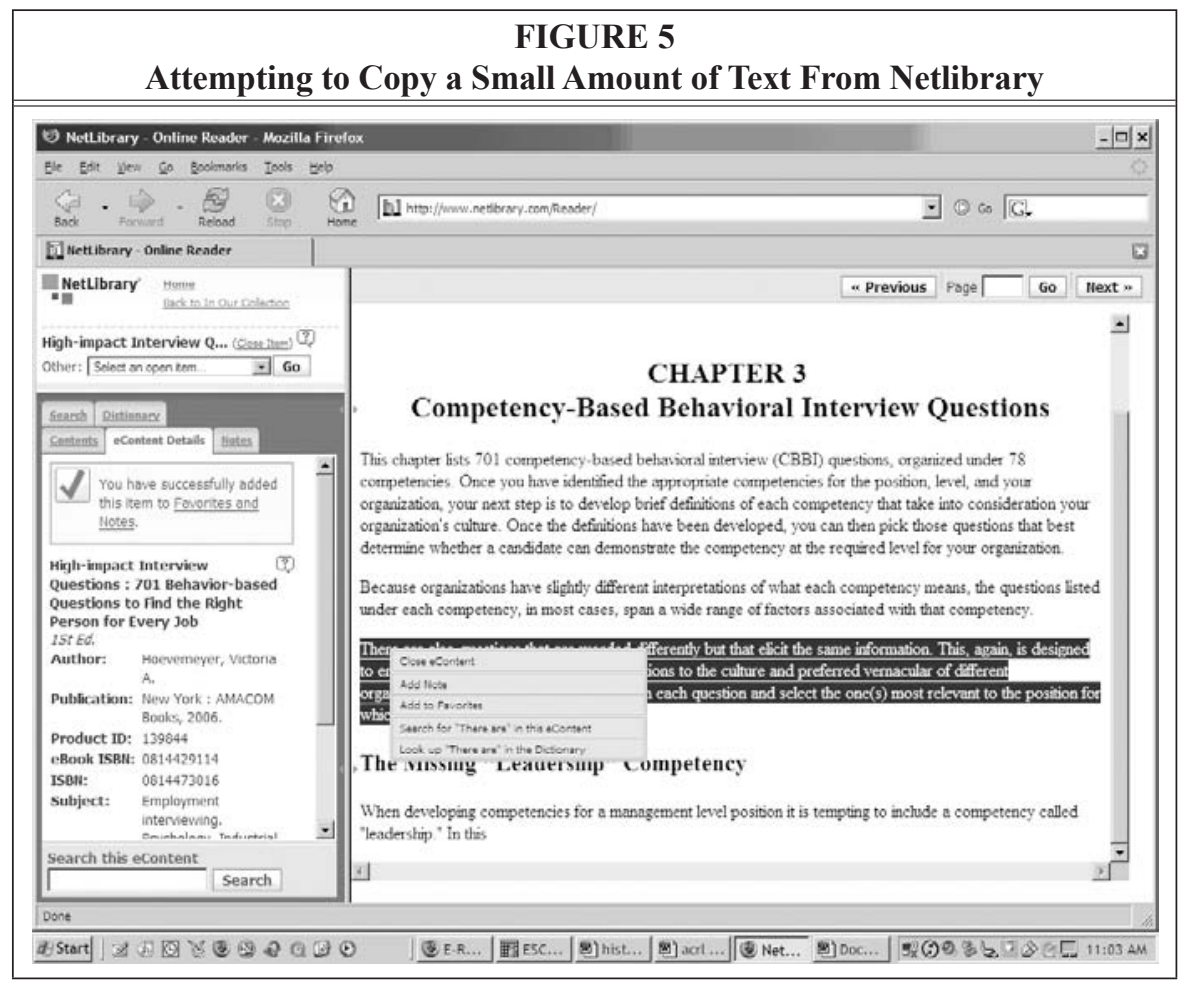


traditional right-click copy feature, likely discourages the copying of text because some users will not realize it is possible to do so.

Most vendors permitted copying of data for personal use. ${ }^{40}$ But copying data tables with formatting was problematic. We tried to copy tables and formatting from the Journal Citation Reports database, the Cochrane Database of Systematic Reviews and Books at Ovid. In these resources, the interface does not advertise any way to copy or save a data table. Highlighting data with the mouse and copying and pasting into a text document resulted in a tab-delimited block of data without formatting. Copied data could, however, be pasted into Excel. Saving the HTML page allowed recreation of the entire HTML page but not the table and its data apart from the page.

We also assessed two networked CDROMs on library workstations (Encyclopedia of Islam and Encyclopedia Judaica). They did allow users to copy and paste text and images into other documents; however, the native interface did not provide users a save button.

\section{Soft Type 5: Restriction by}

Decomposition ${ }^{41}$

The hybrid nature of many HTML eresources complicates use functions such as saving and e-mailing because of the large amalgam of files and file types associated with an HTML document. Take, for example, the overview article about Acne in the health sciences resource Stat!Ref shown in figure 6. Stat!Ref contains a very clear "Print" feature, but no "Save" or "E-mail" feature. Saving the article through browser or operating system functionality is potentially confusing because saving the page decomposes it into an amalgam of subfolders and subfiles. Attempting to view the article locally, transfer it to a memory stick, or e-mail it to a

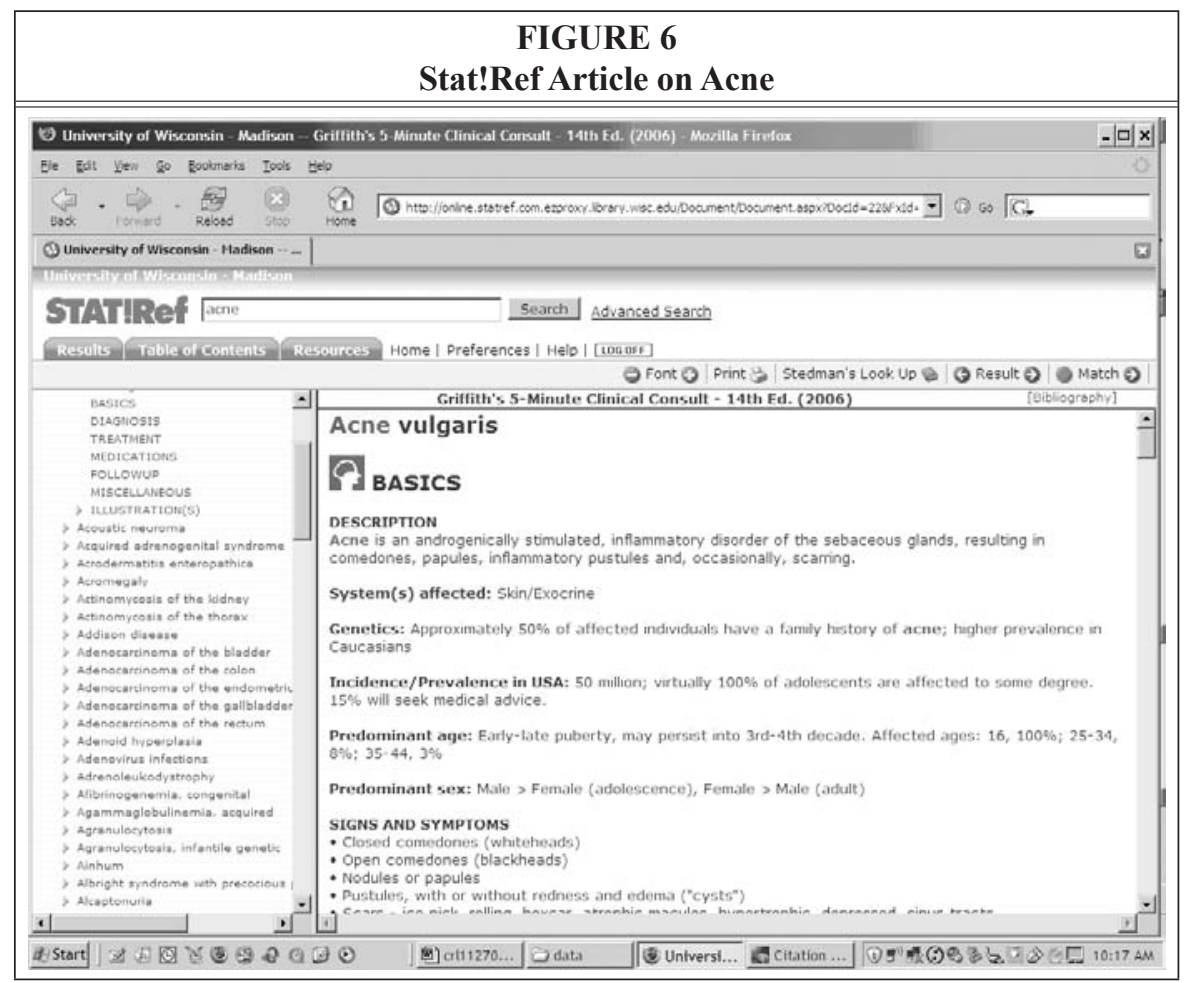




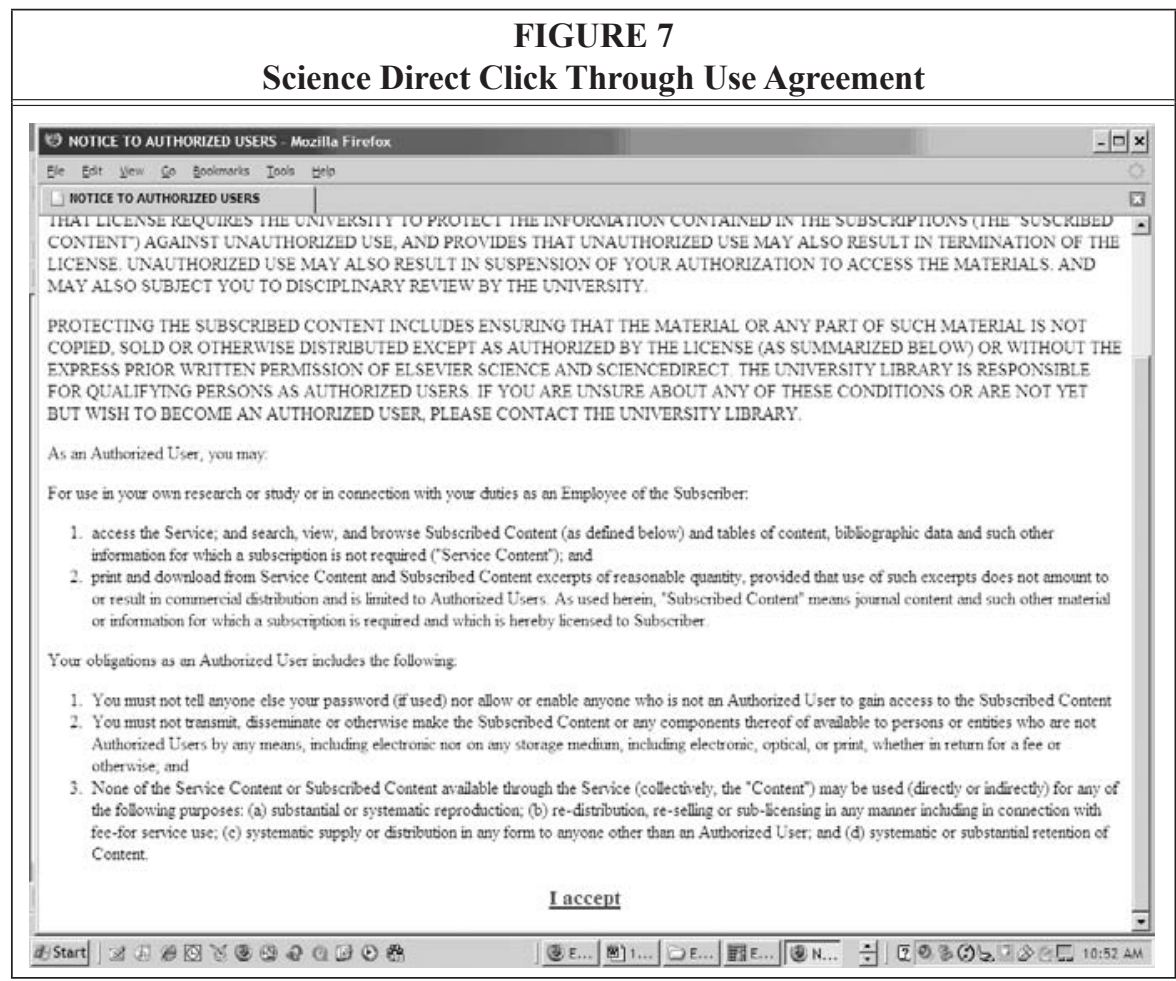

colleague requires that the user be able to reassociate numerous files, or decide which files contain needed text and which can be discarded. This may be particularly problematic when a novice user needs to transfer or e-mail text and associated image files.

The Stat!Ref "User Responsibilities" document gives explicit permission for personal copying, saving, and printing; however, it seems to disallow e-mailing under a clause that prohibits transmission. ${ }^{42}$ Therefore, while the Stat!Ref resource allows saving, the HTML nature of the materials makes it difficult for the average user to reuse or transfer saved documents for personal use. Further, the file format likely reinforces the Stat!Ref prohibition on e-mailing documents.

Soft Type 6: Restriction by Warning In some instances, notices or end user licenses contained within a resource proclaim use limitations. In one case, notices were accompanied by threats of disciplinary action. The Science Direct click through end user statement in figure 7 suggests that misuse may result in disciplinary review by the user's university.

Other examples included use-discouraging messages in the interface or popups that occur when a use is attempted. For example, in the Oxford Dictionary of National Biography (Oxford NBD), clicking on the image brings up a popup that warns "You are not permitted to download or reproduce this image from the Oxford DNB Online web site."

Review of the public license of the Oxford DNB suggests that users do not have permission to save images regardless of whether that use constitutes fair use. "No illustration from the web site may be copied, modified, published or broadcast, or otherwise distributed." 43 Using the "right-click" operating system feature of Windows to save the image produced yet another copyright warning message (see figure 8 ). The Science Direct and Oxford DNB warnings reinforce the 


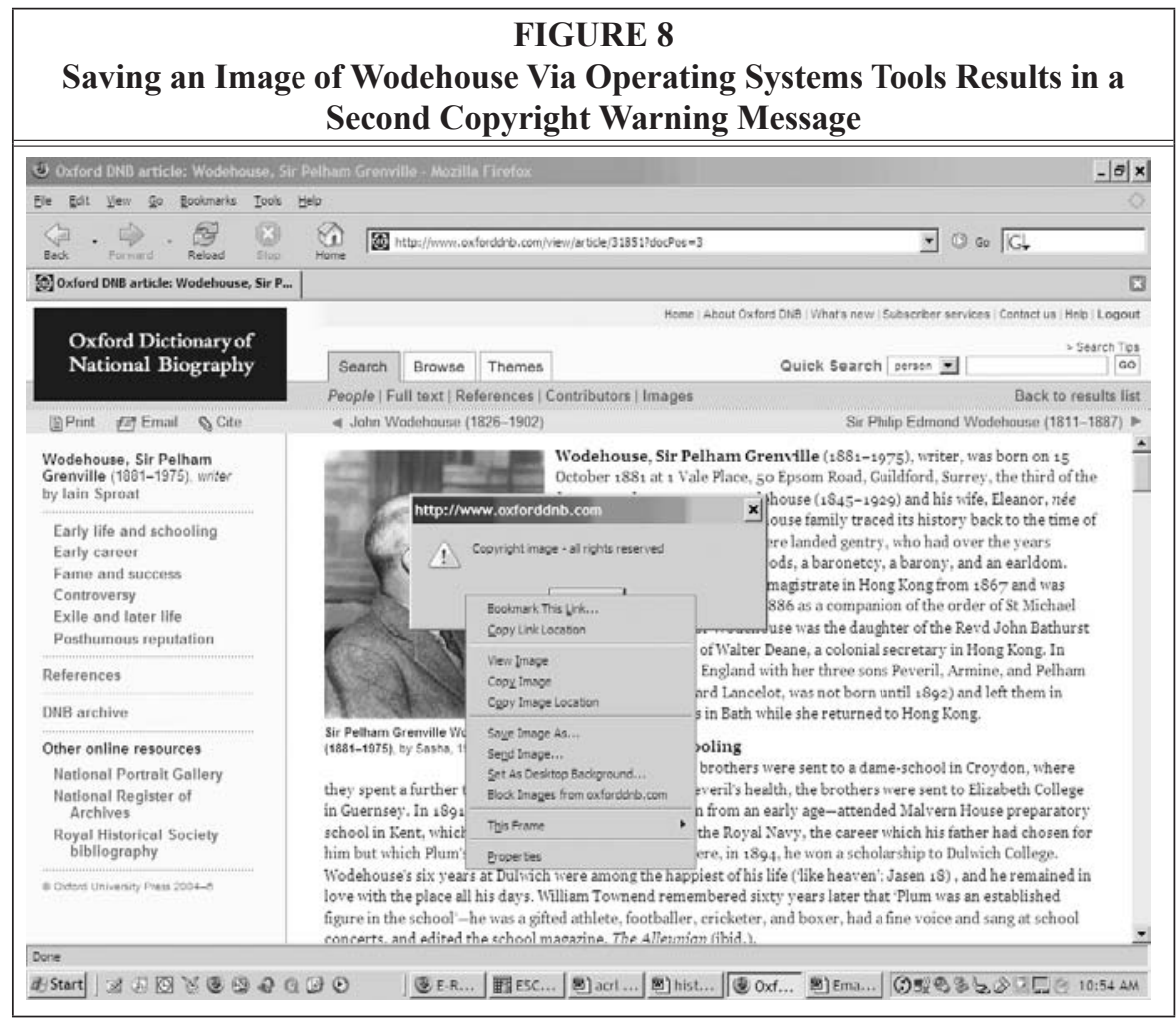

terms of their acceptable use statements by discouraging certain uses, but neither strictly prevents them.

\section{Hard Restrictions}

In contrast to soft restrictions, hard restrictions strictly prevent use. The only hard restriction we observed in our sample was blocked copy and paste functionality. In addition, we learned through interviews of two other products with hard restrictions that employed secure container TPM, and we learned of two instances where hard restriction TPM had been implemented but then removed.

Hard Type 1: No Copy and Pasting of Text

We observed a few examples, mostly contained in historical collections, of resources that did not allow the user to copy and paste text (for example, America's Historical Newspapers, Early American Imprints, Gerritsen Collection, Times
Digital Archive, ECCO). The affected collections were all digitized versions of microfiche. While the fiche did have OCR that permitted keyword searching, the interface did not allow access to the OCR for copying and pasting. However, the user could copy, save, and print images of the text.

For example, in the America's Historical Newspaper collection article seen in figure 9, no mechanism is provided to view the OCR of the document in order to copy and paste the text.

We were unable to find a use statement for America's Historical Newspapers, so it was not possible to compare the restriction with the stated terms of use. It may be that the OCR within these resources was too dirty to permit cutting and pasting of any real utility.

Hard Type 2: Secure Container Technological Protection Measures

We found no examples of secure con- 
tainer TPM in our sample; however, interviews pointed to two examples outside our sample. The first, ARTstor, has a TPM that controls the use of high resolution images downloaded from the library. The ARTstor terms of use are generous, allowing for TPM free copies of lower resolution images, saving of images, and even incorporation of images into other works. In the second example, the Society for Automotive Engineers (SAE) digital library began to implement a secure container TPM in 2007, but pulled back and eventually removed the TPM from the academic library product. SAE information about the original TPM described very limiting use terms. The proposed TPM would have tied downloaded documents to one machine. Users would only have been permitted to view documents and print one copy because the TPM would not have allowed saving, e-mailing, or otherwise transferring copies. SAE's TPM based use restrictions were tied to a "pay per download" pricing scheme. ${ }^{44}$

Interviews also pointed to two other instances where vendors had implemented a secure container TPM, but then withdrew it after customer protests that the TPM violated the terms of their license agreements. Librarians described how Elsevier had implemented a TPM on ebooks in the Referex Engineering Village, and how Knovel had implemented a TPM on one title within the Knovel Engineering \& Scientific Online References.

\section{Analysis \& Discussion Methodological Issues and Study Limitations}

Study results are subject to several limitations. First, the results are limited by the sample of licensed resources employed. The sample does not include all possible licensed resources from engineering, health sciences, and history. It likely includes popular and common resources

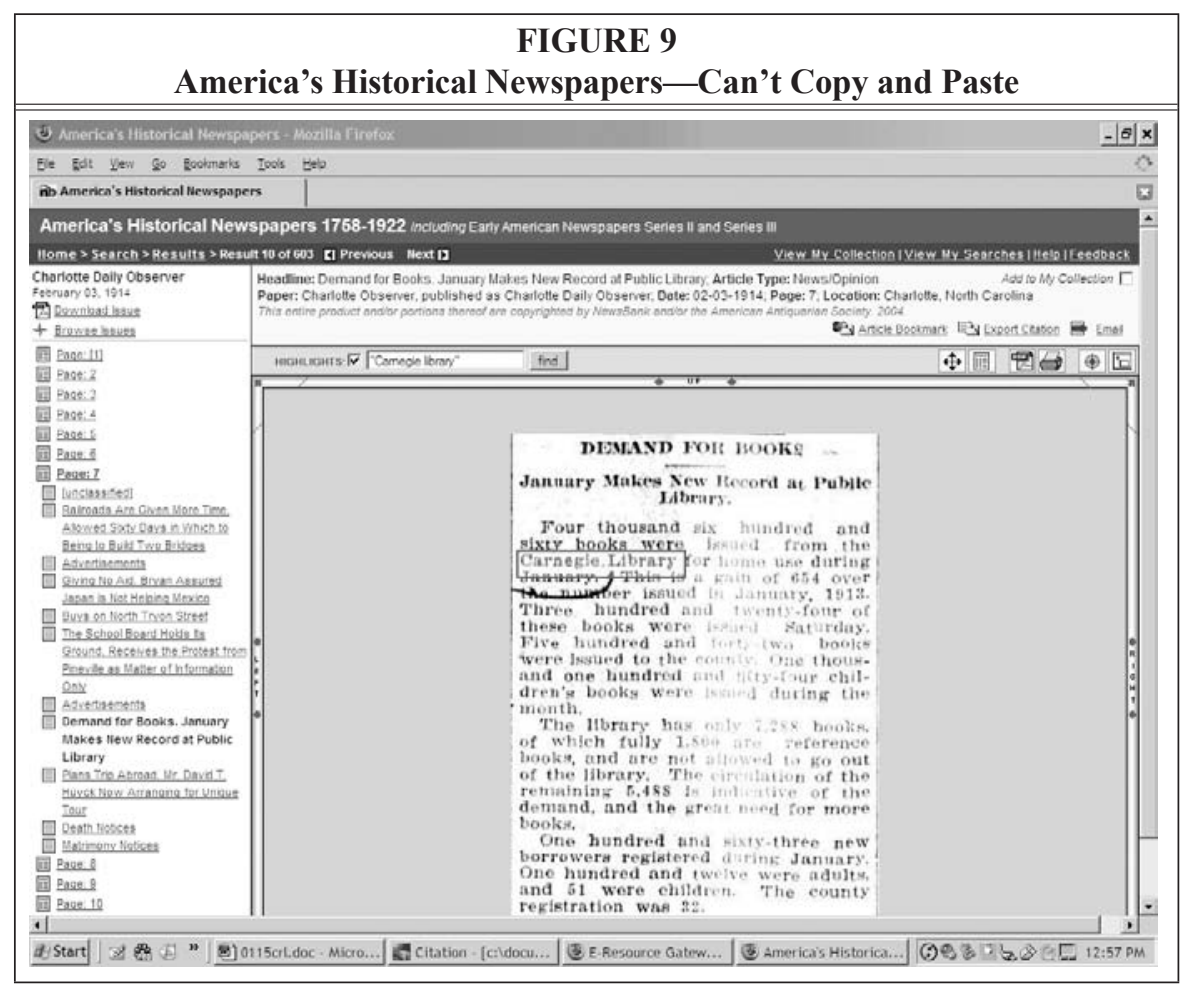


from those areas, but may exclude niche specialty resources and very expensive resources. Further, more research is needed to assess what types of use restrictions exist in licensed resources from other fields; for example, the data did not include the audio streaming tools often employed by licensed music resources.

Another problem is that because licensed resources are maintained on vendor servers (except for networked CD-ROMs), it was impossible to control for interface or service changes during the course of the study. Further, given the malleable nature of licensed electronic resources, our results only represent a snapshot of restrictions found at one point in time. Moreover, the relationship between browser plug ins, operating systems, and vendor interfaces is constantly changing and may influence the observed use restrictions. Finally, the results are also bound by the methodology of the assessment protocol. Different assessment criteria and methods might have uncovered different use restrictions.

Importantly, we have no explanations for most of the observed use restrictions. We do not know if most observed restrictions stem from a desire to curb use, placate content owners, bad interface design, or technological limitations. We did not seek vendor explanations of the observed use restrictions; therefore it is not possible to draw conclusions about vendor intentions.

Several methodological lessons from the study are worth noting. First, we found that random sampling of resources was not effective in identifying products containing TPM. Because resources with TPM are unattractive, librarians often choose not to license them, thereby removing them from a population. For example, the librarians at the case site chose to cancel their Society of Automotive Engineers Digital Library subscription because of its TPM. Future research should identify TPM through interviews or by observing subject specialty library publications, listservs, and blogs.
Moreover, the examples described by librarians suggest that restrictions may vary within a resource. Random sampling of titles within a resource could miss TPM if they are contained on only one title. Use of interviews or list/blog observations would also provide a way to find out which, if any, titles contained notable restrictions.

\section{TPM in Scholarly Publishing}

The question of whether or not vendors of scholarly information will employ TPM on licensed resources remains open, but results support the contention that few hard, secure container TPM have survived long in academic library markets. Several vendors have implemented, and then withdrawn, hard TPM (i.e., Knovel, Referex, and the SAE Digital Library). We don't know why the Knovel and Referex TPM implementations failed; however, a separate manuscript describes and analyzes the failed SAE implementation. ${ }^{45}$ Within the publishing industry, critiques of TPM have pointed to end user and librarian acceptance issues and high costs as a reason to avoid TPM. ${ }^{46}$ Of the described resources, only ARTstor has maintained its TPM. ${ }^{47}$

The study results also show that the scholarly literature is not free from use restrictions. If one shifts focus from secure container TPM to use restrictions in general, then use restrictions are common.

This raises the issue of the level of attention paid in the library professional literature to TPM in comparison to the soft use restrictions described in this paper. While articles on TPM have appeared regularly in the library literature, it is more difficult to find systematic studies of use restrictions in licensed resources. This may stem from license clauses that prohibit "testing" of resources, the large number of licensed resources to test, lack of manpower to undertake testing, and the ever changing nature of vendor interfaces. Tracking use restrictions of any significant number of resources would require considerable resources. Further, 
some of the use restrictions may not qualify as egregious enough to track.

One concern is that the library community has already accepted many of the soft use restrictions identified in this paper. If librarians do nothing to protest these restrictions, they give implicit consent that the use restrictions are acceptable. Librarians have advocated for other interface issues like accessibility; they should also advocate for removal of use restrictions, or encourage new vendors to offer competing restriction-free products. Many (but not all) of the use restrictions described in this paper arguably are TPM - they control how patrons use information products. They deserve more attention and advocacy work than they currently receive from the library community.

It is unrealistic for any one library to keep track of the use restrictions present in the multitude of resources they license. A more realistic solution might be a shared knowledge base of vendor interfaces and known use restrictions. Libraries could take responsibility for tracking interface issues associated with a particular vendor or platform, and share that knowledge so that all libraries would be informed of potentially objectionable use restrictions, interface changes, or even interface improvements. Shared reporting systems already exist within some larger

\begin{tabular}{|c|c|c|c|}
\hline \multicolumn{4}{|c|}{$\begin{array}{c}\text { TABLE } 2 \\
\text { Summary of Use Restriction Types and } \\
\text { Examples from the Data }\end{array}$} \\
\hline & Definition & $\begin{array}{l}\text { Restriction } \\
\text { Subtype }\end{array}$ & $\begin{array}{l}\text { Observed Use } \\
\text { Restrictions }\end{array}$ \\
\hline \multirow[t]{6}{*}{$\begin{array}{l}\text { Soft } \\
\text { Restrictions }\end{array}$} & \multirow{6}{*}{$\begin{array}{l}\text { Interface or server side } \\
\text { configuration of hardware } \\
\text { and software that may } \\
\text { discourage certain uses } \\
\text { such as saving, printing } \\
\text { multiple pages, e-mailing. } \\
\text { Importantly, desired use } \\
\text { may be achieved via } \\
\text { operating system or browser } \\
\text { functionalities; however, it } \\
\text { may be inconvenient. }\end{array}$} & Extent of use & $\begin{array}{l}\text { Page print limits, PDF } \\
\text { download limits; data } \\
\text { export limits; suspicious } \\
\text { use tracking. }\end{array}$ \\
\hline & & Obfuscation & $\begin{array}{l}\text { Needing to select items } \\
\text { before use options be- } \\
\text { come available. }\end{array}$ \\
\hline & & Omission & $\begin{array}{l}\text { Not providing buttons or } \\
\text { links to enact uses. }\end{array}$ \\
\hline & & Decomposition & $\begin{array}{l}\text { Saving document results } \\
\text { in many files, making } \\
\text { recreating or e-mailing } \\
\text { the document difficult. }\end{array}$ \\
\hline & & Frustration & $\begin{array}{l}\text { Page chunking in e- } \\
\text { books. }\end{array}$ \\
\hline & & Warning & $\begin{array}{l}\text { Copyright warnings, end- } \\
\text { user licenses on startup. }\end{array}$ \\
\hline \multirow[t]{2}{*}{$\begin{array}{l}\text { Hard } \\
\text { Restrictions }\end{array}$} & \multirow{2}{*}{$\begin{array}{l}\text { Combinations of hardware } \\
\text { and software that strictly } \\
\text { control or disallow direct } \\
\text { or subsequent use actions in- } \\
\text { cluding saving, printing, or } \\
\text { e-mailing despite operating } \\
\text { system or browser function- } \\
\text { alities. }\end{array}$} & $\begin{array}{l}\text { Restricted copy } \\
\text { and paste OCR }\end{array}$ & $\begin{array}{l}\text { OCR exposed for search- } \\
\text { ing, but not for copying } \\
\text { and pasting of text. }\end{array}$ \\
\hline & & $\begin{array}{c}\text { Secure } \\
\text { container TPM }\end{array}$ & $\begin{array}{l}\text { Use rights vary by } \\
\text { resource. }\end{array}$ \\
\hline
\end{tabular}


library systems; however, they tend not to focus on use restrictions.

One contribution of this paper is to provide a vocabulary for talking about use restrictions. It is important that librarians be able to identify and talk about the different types of use restrictions they perceive as creating usability issues. Being able to name something you don't like may be the first step to getting rid of it. Table 2 summarizes the framework of use restrictions developed from the data.

While this study identifies the soft use restrictions common in scholarly licensed resources, more research is needed to identify the technical, business, and disciplinary circumstances under which vendors employ various types of restrictions. For example, why were HTML format resources and concomitant decomposition problems very common in the health sciences field? Why do some e-book vendors employ larger chunks while others continue with page view chunks?

\section{Effects-Shaping Use Expectations}

Some use restrictions matter more than others. HCI research is needed to determine to what extent use is actually curtailed by the soft restrictions we identified. Soft restrictions could serve as highly effective "soft TPM," restricting use without creating the negative controversy of a secure container TPM. Or, they could merely be annoyances that most users figure out how to workaround.

Given our limited data, we can only speculate about how use expectations will change over time, and whether the soft restrictions described in this paper will become the taken for granted norm. ${ }^{48}$ One can hope that user creativity, combined with effective license negotiations by librarians, will shape the future uses permitted by licensed resources, rather than the other way around.

\section{Conclusion}

As part of an exploration of TPM use in the academic publishing industry, this paper described the use restrictions found in a sample of licensed scholarly digital resources from the fields of engineering, health sciences, and history/art history. Analysis distinguished between hard restrictions that strictly prevent use, and soft restrictions that make certain uses inconvenient or difficult to achieve. The paper identifies six types of soft restrictions: extent of use, obfuscation, omission, decomposition, frustration, and warning. Results found only one example of a secure container style TPM employed by a vendor, but pointed to three cases of vendor TPM trials that failed.

The paper describes numerous examples of soft use restrictions employed by vendors, and argues that some act as TPM, though they are different from the "secure container" TPM employed in the music and movie industries. The paper argues that soft use restrictions deserve more attention from the library community, and that librarians should not accept these restrictions as the natural order of things. More HCI testing is needed to find out which soft restrictions actually block desirable uses, and more advocacy work is needed to persuade publishers to remove these use restrictions.

\section{Notes}

1. This study was funded in part by Institute for Museum and Library Services Laura Bush 21st Century Research Grant RE-04-06-0029-06 and the American Library Association Carroll Preston Baber Award. Pretesting was supported by the 2005 American Library Association Samuel Lazerow Fellowship. An earlier version of this paper appeared in the 2007 Proceedings of American Society for Information Science and Technology. This version includes more data and updated analysis. The author thanks Ian Benton for his research assistance and all the study participants for their time and insights. This paper benefited from feedback from Susan Barribeau, Michelle Besant, Deborah Helman, Christine Kline, Chatherine Arnott Smith, Stephen Paling and Greg Downey. 
2. This narrower term of TPM is preferable because DRM refers more broadly to the management of rights from both a licensor and licensee perspective.

3. NetLibrary. Frequently Asked Questions About PDF Books. 2002. Available online from http:// a1835.g.akamai.net/7/1835/276/1ca66b46d8d81e/extranet.netlibrary.com/extranet/publisher/specifications/documents/PDF_FAQ.pdf. [Accessed 10 December 2006]

4. C. Lynch, Colleges, Codes, and Copyright: The Impact of Digital Networks and Technological Controls on Copyright and the Dissemination of Information in Higher Education. June 10-11. (University of Maryland: University of Maryland: University College, 2004); U. Riehm, "Science Can't Accept Technical Barriers of Content Use!" INDICARE Monitor (Nov. 2005): 3 pages. Available online at http://www.indicare.org/tiki-read_article.php?articleId=129 [Accessed April 2006].

5. Unless that uncontrolled use involves large-scale, systematic sharing between institutions such that the recipient institution avoids subscribing.

6. Orwat, Carsten "Digital Rights Management in Public Science: Report on the 4th INDICARE Workshop Brussels, Belgium" (INDICARE Project, 2006). Available online http://www.indicare. org/tiki-download_file.php?fileId=176 [Accessed April 2006].

7. Orwat, "Digital Rights Management and Public Science"; Rosenblatt, B. DRM and Research Libraries: Common Ground? Against the Grain 15, no. 5 (2003): 22-23.

8. P. Hernon and P. Calvert, "E-service Quality in Libraries: Exploring Its Features and Dimensions," Library and Information Science Research 27 (2005): 377-404; H.I. Xie, "Evaluation of Digital Libraries: Criteria and Problems from Users' Perspectives, Library and Information Science Research 28 (2006): 433-52.

9. Xie, "Evaluation of Digital Libraries."

10. P.S. Adler, Hearing on Fair Use: Its Effects on Consumers and Industry, Subcommittee on Commerce, Trade, and Consumer Protection, U.S. House of Representatives Committee on Energy and Commerce. November 2005; G. Agnew and M. Martin, "Digital Rights Management: Why Libraries Should Be Major Players," in Bowker Annual: Library and Book Trade Almanac, ed. D. Bogart (Medford N.J.: Information Today, 2003): 267-78; K. Coyle, Rights Expression Languages: A Report for the Library of Congress (Washington, D.C.: Library of Congress, 2004)

11. Agnew and Martin, "Digital Rights Management"; E.W. Felten, "A Skeptical View of DRM and Fair Use," Communications of the ACM 46, no. 4 (2003): 56-59; J. Cohen, "The Challenge of Digital Rights Management Technologies," in The Role of Scientific and Technical Data and Information in the Public Domain: Proceedings of a Symposium (Washington, D.C.: National Academy Press, 2003), 109-16; L. Lessig, The Future of Ideas (New York: Random House, 2001); L. Lessig, Free Culture: How Big Media Uses Technology and The Law to Lock Down Culture and Control Creativity (New York: Penguin Press, 2004).

12. C.W. Bailey, "Strong Copyright + DRM + Weak Net Neutrality = Digital Dystopia," Information Technology and Libraries 25, no. 3 (Sept. 2006): 116-27.

13. Library Copyright Alliance. Library-Related Principles for the International Development Agenda of the World Intellectual Property Organization. (Washington DC: Library Copyright Alliance, 2006) Available online http://www.librarycopyrightalliance.org/wipo.htm [Accessed December 11, 2006]; European Bureau of Library, Information and Documentation Associations. EBLIDA Position on Digital Rights Management Systems. (The Hague: EBLIDA: 2003). Available online at www.eblida. org. [Accessed October 21, 2005].

14. The study does not consider access controls that limit use to authorized users.

15. T. Striphas and K. McLeod, "Strategic Improprieties: Cultural Studies, the Everyday, and the Politics of Intellectual Property," Cultural Studies 20, no. 2 (May 2006): 119-44; S. Vaidhyanathan, "Afterword: Critical Information Studies, A Bibliographic Manifesto," Critical Studies 20, no. 2-3 (2006): 292-315.

16. W. Bijker, Of Bicycles, Bakelites and Bulbs: Toward a Theory of Socio-Technical Change (Cambridge, Mass.: MIT Press, 1995).

17. T. Gillespie, "Designed to 'Effectively Frustrate': Copyright, Technology and the Agency of Users," New Media E Society 8, no. 4 (2006): 651-69. Gillespie, T. Wired Shut: Copyright and the Shape of Digital Culture. (Cambridge, MA: MIT Press, 2007); Samuelson, “DRM \{and, or, vs.\} the Law," Communications of the ACM 46, no. 4 (2003): 41-45.

18. L. Lessig, Code and Other Laws of Cyberspace (New York: Basic Books, 1999); M. Nentwich, Cyberscience: Research in the Age of the Internet (Vienna: Austrian Academy of Sciences Press, 2003).

19. Lessig, Free Culture

20. Note that licenses provide protection from the imposition of new TPM that impose use constraints not addressed within a license. Libraries can refuse to sign new licenses that require use of TPM. For example, in 2006-2007 many engineering librarians refused to renew licenses for the Society for Automotive Engineers (SAE) database that included new TPM requirements.

21. Adler, Hearing on Fair Use.

22. Coyle, Rights Expression Languages: A Report for the Library of Congress. 
23. We collected data on access constraints, but we do not report them here due to space constraints and because the results were not novel. Readers interested in access controls may see Agnew or Curtis for an overview.

24. L.E. Harris, Licensing Digital Content: A Practical Guide for Librarians (Chicago: American Library Association, 2002); A. Braid, "The Use of Digital Rights Management in Document Supply," INDICARE Monitor 2 (May 2005) 1-10; Center for Democracy and Technology, Evaluating DRM: Building a Marketplace for the Convergent World (Washington, D.C.: Center for Democracy \& Technology, 2006); Intrallect Inc., Digital Rights Management: Final Report for the JISC (Linlithgow, U.K.: Intrallect Inc., 2004); M. Jackson and A. Shah, Australian Information Security Workshop 2005, "The Impact of DRMs on Personal Use Expectations and Fair Dealing Rights," in Conferences in Research and Practice for Information Technology. 44 (2005): 1-8.

25. W.Y. Arms, Digital Libraries (Cambridge, Mass.: MIT Press, 2000).

26. We did not retain documents over long periods to assess whether or not they expired. Lack of secure container TPMs and lack of security features on downloaded documents suggested that the documents we saved would not expire.

27. Cohen, "The Challenge of Digital Rights Management Technologies"; M. Nentwich, "Cyberscience: Modelling ICT-Induced Changes of the Scholarly Communication System," Information, Communication \& Society 8, no. 4 (2005): 542-60.

28. Arms, Digital Libraries; Rosenblatt, B. Trippe, and S. Mooney, Digital Rights Management: Business and Technology (New York: M\&T Books, 2002); K. Coyle, "The Technology of Rights: Digital Rights Management," 2003, available online from www.kcoyle.net/drm_basics.pdf [accessed 12 December 2006].

29. The sampling universe was composed of lists of electronic resources relevant to each subject area developed by subject area specialist librarians and confirmed or modified during interviews with librarians.

30. See Appendix A for the titles of the resources assessed within each discipline area.

31. Resource use comported with CONFU guidelines for fair use of materials for e-reserves.

32. Within each resource, we selected three sections of content for review. Sections of content included whole articles within an article database, book chapters within an e-book, encyclopedia entries within a reference resource, and individual letters within a collection of digitized letters. Content selections were driven by the assessor's personal interests and identified by keyword searches or browsing. Results that did not contain images or that were too short were not used. If a search generated numerous results, then the assessor chose one result from the first few results, one from in the middle of the results, and one from near the end. If the results were arranged chronologically, one new result was chosen, one older result was chosen, and one very old result was chosen.

33. Bailey "Strong Copyright + DRM + Weak Net Neutrality = Digital Dystopia."

34. Blackwell Publishing, "Update on IP Blocking," Blackwell Publishing News. April/May 2006. Available online from www.blackwellpublishing.com/librarians/newsletter_html/March06Newsletter30.htm\#8. [Accessed 12 December 2006].

35. Gale Publisher (ECCO) Terms and Conditions: Copyright and Limitations on Use reads: "The subscribing institutes ('Customer') and their authorized users, may make a single print, nonelectronic copy of a permitted portion of the content for personal, non-commercial, educational purposes only."

36. National Academy Press Terms of Use Statement reads: "No part of these pages, either text or image may be used for any purpose other than personal use." Net Library Terms of Use Statement reads: "You may not copy the Copyrighted Material or engage in any other acts inconsistent with the principles of copyright protection and fair use, as codified in 17 U.S.C. Sections 106-110, without obtaining the express written permission of NetLibrary and the copyright owner." We were unable to locate a Taylor and Francis acceptable use statement on the CRC Web site.

37. The book employed in the example is High Impact Interview Questions: 701 Behaviorbased Questions to Find the Right Person for Every Job. Hoevermeyer, V.A. (2006) New York: AMACOM Books.

38. Javascript, as well as several off the shelf software products, can be used to disabling right click copy features or create alternative right click menus or copyright notices.

39. "You may not copy the Copyrighted Material or engage in any other acts inconsistent with the principles of copyright protection and fair use, as codified in 17 U.S.C. Sections 106-110, without obtaining the express written permission of NetLibrary and the copyright owner. In the event of any permitted copying, redistribution or publication of copyrighted material, no changes in or deletion of author attribution, trademark legend or copyright notice will be made." NetLibrary Terms of Use Agreement Point 3C.

40. For example, the ISI Web of Knowledge Acceptable Use Policy reads: “Copying/duplicating the Licensed Product or creating subsets or derivative databases from the Database, except 
for personal use only."

41. In earlier related works we refer to this type of TPM as "TPM by Polyglot."

42. The Teton Data Systems (Stat!Ref) User Responsibilities Document reads: "You may copy, save, and print information from the System. Except as otherwise expressly provided in this UR, you may not, in whole or in part: (a) copy, distribute, transmit, display, perform, reproduce, publish, modify, rewrite, create derivative works from, transfer or sell any material contained in System."

43. Oxford Dictionary of National Biography Copyright Statement-Available online from http://www.oup.com/oxforddnb/legal/. [Accessed November 2006]. The use restrictions on images likely stems from ODNB's lack of ownership of the images.

44. Library Journal Online. "MIT Automotive Engineering Faculty Say No to DRM." (New York: Library Journal, March 28 2007) http://www.libraryjournal.com/article/CA6428576.html [Accessed April 6 2007]; K.R. Eschenfelder, B. Walden, "A Tale of Two DRM: The Co-Construction of Access and Use Rights for Licensed Digital Resources" (In Press in the Proceedings of the Annual Meeting of the American Society for Information Science and Technology, 2008)

45. Eschenfelder "A Tale of Two DRM."

46. Orwat, "Digital Rights Management in Public Science"; INDICARE Project (2006) Content Providers' Guide to Digital Rights Management. http://www.indicare.org/tiki-download_file. php?fileId=175 [Accessed December 2006]

47. Eschenfelder "A Tale of Two DRM." Law."

48. Bijker, Bicycles, Bakelites and Bulbs; Gillespie, Wired Shut; Samuelson, "DRM \{and,or,vs.\} the

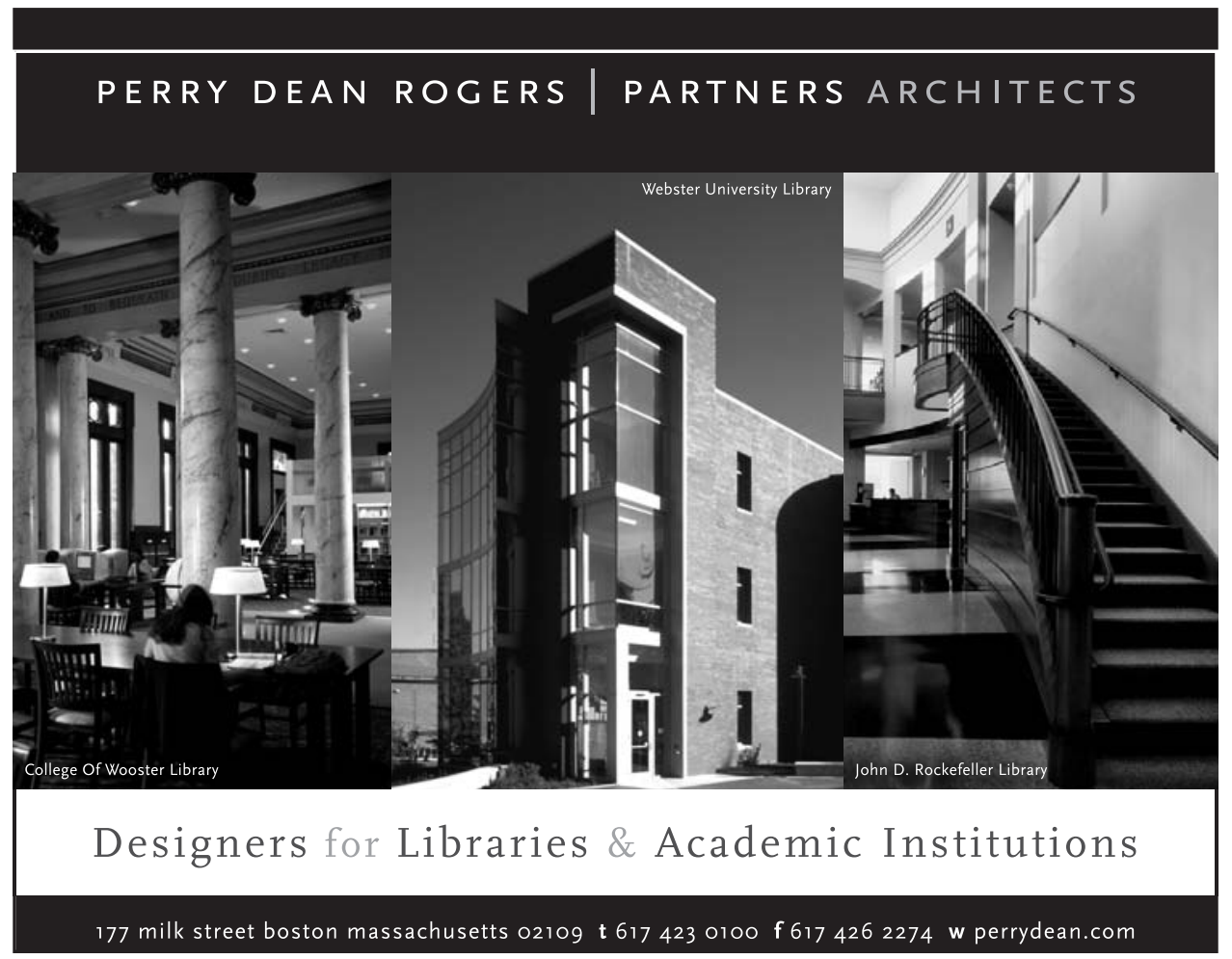




\section{Appendix A \\ Resources Included in Study}

\section{Engineering}

1. ACM Digital Library

2. Advances in Biochemical Engineering/Biotechnology

3. American Chemical Society Publications and Journals

4. American Institute of Physics (AIP) Online Journal Publishing Services

5. American Meteorological Society

6. Applied Science Full Text

7. ASCE Digital Library /Scitation

8. Business Source Elite

9. CRC Handbook of Chemistry and Physics

10. Electrochemical Society (ECS) Publications

11. ENGnetBase Engineering Handbooks Online

12. GeoRef

13. IEEE Explorer

14. Ingenta Connect

15. Institute of Physics Electronic Journals Collection (IOP)

16. Kirk-Othmer Encyclopedia of Chemical Technology

17. KNOVEL

18. Lecture Notes in Computer Sciences

19. Materials Research Society (MRS) Proceedings Library

20. Royal Society of Chemistry Online Journals

21. SAE Digital Library

22. Safari Tech Books Online

23. Science Direct

24. Society for Automotive Engineers Digital Library

\section{Health Sciences}

1. American College of Physicians Journal Club (ACP)

2. Annual Reviews

3. Beilstein Crossfire Organic and Gmelin Inorganic/Organometallic Chemistry Database

4. Books 24/7

5. Books at Ovid Products

6. Business and Industry

7. CINAHL

8. CINAHL Plus

9. Clinical Reference Systems

10. Cochrane Reviews

11. DARE Database of Abstracts of Reviews of Effects

12. Early American Imprints

13. Education Full Text

14. Elsevier/Harcourt Health Journals (Science Direct)

15. Entrez (NCBI)

16. Health and Psychosocial Instruments

17. Health and Wellness Resource Center

18. Inforretriever/InfoPOEMS

19. Karger Journals

20. LEA Online 
21. MD Consult

22. Micromedex

23. Proteome Bioknowledge Library

24. Sage Journals

25. SciFinder Scholar

26. SpringerLink

27. Stat! Ref

28. Up to Date

\section{History/Art History}

1. Access UN

2. Art Full Text

3. ARTstor

4. Bibliography of the History of Art

5. British and Irish Womens Letters and Diaries

6. Early Encounters in North America

7. Early English Books Online (EEBO)

8. Eighteenth Century Collections Online (ECCO)

9. Encyclopedia Islam

10. Encyclopedia Judaica

11. Gerristen Collection

12. JSTOR

13. Making of America at Michigan

14. NetLibrary

15. North American Immigrant Letters, Diaries and Oral Histories

16. North American Women's Letters and Diaries Colonial to 1950

17. Oxford Dictionary of National Biography

18. Oxford English Dictionary

19. Proquest Digital Dissertations

20. Proquest Research Library

21. Social Sciences Full Text

22. Times (London) Digital Archives

23. Ulrichs

24. Waterloo Dictionary of English Newspapers \& Periodicals (1800-1900)

25. Web of Knowledge 


\section{TAP into a New World OF ONLINE REVIEWS}

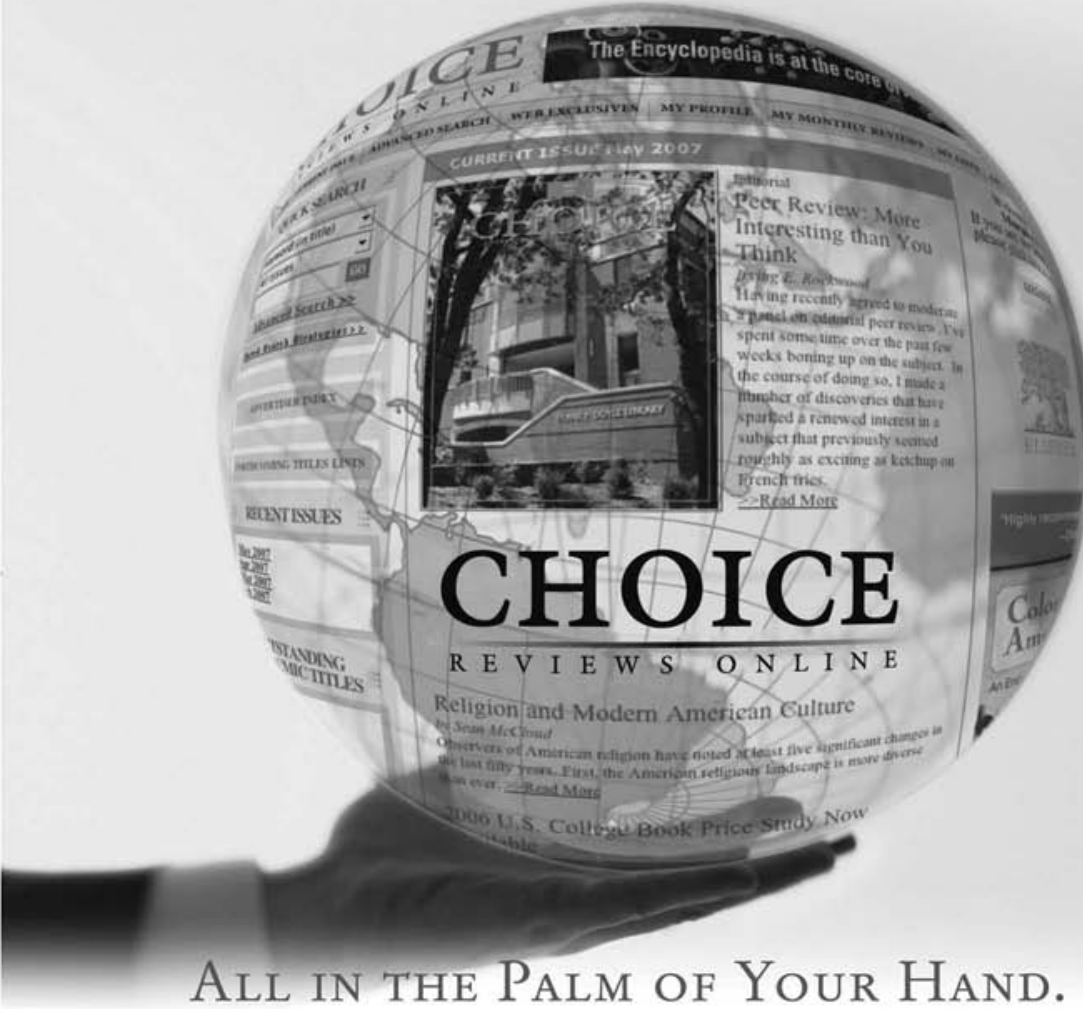

- Access to over 120,000 Choice Reviews

- Advanced Search Capabilities

- Enhanced Customization Possibilities

- Download, Print, or E-mail Search Results
- Exclusive Online Content

- Comprehensive Content

- User-Friendly Interface

To learn more about Choice Reviews Online, visit us at booth \#2417 at the ALA Annual Conference in Anaheim, CA. June 28 - July 1

To sign up for free 60-day trial, visit http://www.cro2.org/

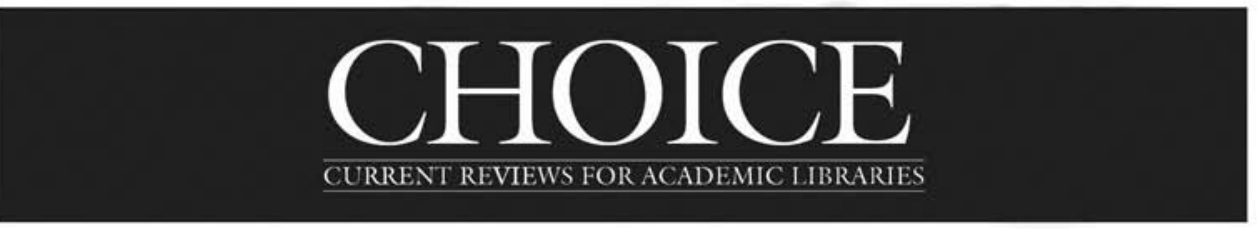

